\title{
Adipositas-assoziierte Lymphödeme - unterschätzt und unterbehandelt
}

\section{T. Bertsch}

Földiklinik Hinterzarten - Europäisches Zentrum für Lymphologie

\section{Schlüsselwörter}

Adipositas, Lymphödeme, multimodales Adipositaskonzept, bariatrische Chirurgie

\section{Zusammenfassung}

Der weltweite Anstieg der Adipositasprävalenz - die WHO spricht bereits von einer Adipositasepidemie - hat auch eine dramatische Veränderung innerhalb der Lymphologie zur Folge. Während "klassisch sekundäre" Lymphödeme nach onkologischen Operationen aufgrund verbesserter OP-Techniken abnehmen, steigt die Zahl von Patienten mit Lymphödemen, die Adipositas-assoziiert, sind exponentiell an. Dieser Wandel im lymphologischen Patientengut wird aktuell noch weitgehend unterschätzt - häufig auch negiert. So ist dieses Thema sowohl auf wissenschaftlichen Kongressen als auch in der Fachliteratur, wenn überhaupt nur marginal repräsentiert. Im klinischen Umgang dominiert vor allem Hilflosigkeit gegenüber diesen Patienten. Dabei ist der pathophysiologische Zusammenhang zwischen Adipositas und Lymphödem inzwischen gut belegt. Adipositas kann Lymphödeme verursachen bzw. bestehende Lymphödeme verschlechtern. Therapiekonzepte, die isoliert das Lymphödem fokussieren, die Adipositas aber als wesentliche Ursache ignorieren, sind daher nicht zielführend. Ebenso kontraproduktiv ist ein therapeutischer Ansatz, der Adipositas mittels Diäten bzw. durch kommerzielle Gewichtsreduktionsprogramme behandelt. Diäten und Gewichtsreduktionsprogramme haben im Lang- zeitverlauf bisher nur eines bewiesen - dass sie scheitern. In der Földiklinik werden adipöse Patienten mit Lymphödemen (auch mit Lipödemen) im Rahmen eines multimodalen Adipositasprogramm behandelt. In diesem Programm werden u. a. internistische, psychologische, ggf. auch adipositaschirurgische und plastisch-chirurgische Therapieoptionen angewandt; die Betreuung der Patienten ist langfristig angelegt. Entgegen Vermutungen ist die Therapie von Patienten mit Adipositas-assoziierten Lymphödemen meist sehr dankbar, dankbar weil Patienten nicht nur eine essenzielle Verbesserung ihres Lymphödemes erfahren, dankbar auch, weil viele Patienten durch die im Rahmen eines adipositaschirurgischen Eingriffes erfolgte Gewichtsreduktion wieder in ein normales, weitgehend gesundes Leben zurückkehren.

\section{Keywords}

Obesity, Lymphoedema, multimodal obesity concept, bariatric surgery

\section{Summary}

A dramatic change within the field of Lymphology has been caused by the worldwide increase in the prevalence of obesity, which the WHO has already termed an obesity epidemic. While "classic secondary" lymphoedema after oncological surgery is decreasing due to improved surgical techniques, the number of patients with obesity-associated lymphoedema

Korrespondenzadresse

Dr. med. Tobias Bertsch

Leitender Oberarzt

Földiklinik GmbH \& Co.KG

Rösslehofweg 2-6

79856 Hinterzarten

Tel. +4976521240

Fax +497652124116

E-Mail: tobias.bertsch@foeldiklinik.de
Obesity related Lymphoedema - underestimated and undertreated

Phlebologie 2018; 47: 75-83

https://doi.org/10.12687/phleb2410-2-2018

Eingereicht: 08. Januar 2018

Angenommen: 28. Januar 2018

English version available at www.phlebologieonline.de is increasing at an exponential rate. That change in the lymphological patient population is still being vastly underestimated and frequently ignored. This topic is, if at all, only marginally represented at scientific congresses and in the literature. Helplessness dominates when dealing with these patients in clinical practice, even though the pathophysiological associations between obesity and lymphoedema have been well established. Obesity can cause lymphoedema, and worsen pre-existing lymphoedema.

Therapy concepts which focus solely on the lymphoedema and ignore obesity as a significant cause are therefore not efficient. Therapeutic approaches which attempt to treat obesity with diets or commercial weight-loss programmes are also counterproductive. Long term studies have proven only one thing with regards to these diets and weightloss programmes so far - that they fail.

At the Foeldi Clinic obese patients with lymphoedema (and lipoedema) are treated with a multimodal obesity programme. In this programme we apply therapeutic options from various fields: internal medicine, psychology, and where required bariatric and plastic surgery. The long-term care of patients is planned and laid out.

Unexpectedly, the treatment of patients with obesity-associated lymphoedema is often very rewarding, because patients experience not only an essential improvement of their lymphoedema but are also able to return to a normal, much healthier lifestyle due to the weight loss, through the use of bariatric surgery for example. 


\section{Einleitung}

Adipositas ist weit mehr als nur „zu viel essen und zu wenig bewegen“!

Die Ursachen von Adipositas sind vielfältig und sehr komplex. Wissenschaftlich evident sind genetische und vor allem auch epigenetische Einflüsse (68-71). Biologische Faktoren wie Stress oder Suchtverhalten können das Gewicht beeinflussen (72, 73). Psychische Erkrankungen wie Depressionen und Essstörungen tragen stark zur Adipositas bei (74). Schließlich spielen auch soziokulturelle Veränderungen eine erhebliche Rolle bei der Entwicklung von Adipositas: beispielsweise Veränderungen des Essverhaltens, der exorbitante Zuckerkonsum (vor allem in Soft Drinks und industriell hergestelltem Essen), ein bewegungsarmer Lifestyle sowie ein Schönheitsideal, das im Untergewichtsbereich angesiedelt ist - und daran eng gekoppelt eine Diätkultur, die oft im Jugendalter schon beginnt $(75,76)$.

Die epidemieartige Ausbreitung von Adipositas (1) ist nicht nur ein medizinisches Problem, die ansteigende Adipositasprävalenz ist auch ein ökonomisches, ein politisches und ein psychosoziales Problem. In Deutschland sind knapp $25 \%$ der Menschen adipös (BMI $\left.>30 \mathrm{~kg} / \mathrm{m}^{2}\right)(2)$, in den USA waren es 2017 bereits $40 \%$ (3).

Adipositas ist mit zahlreichen Folgeerkrankungen verbunden. Im Vordergrund stehen kardiologische, endokrinologische, orthopädische aber auch psychische Erkrankungen, die eng mit Adipositas in Zusam-

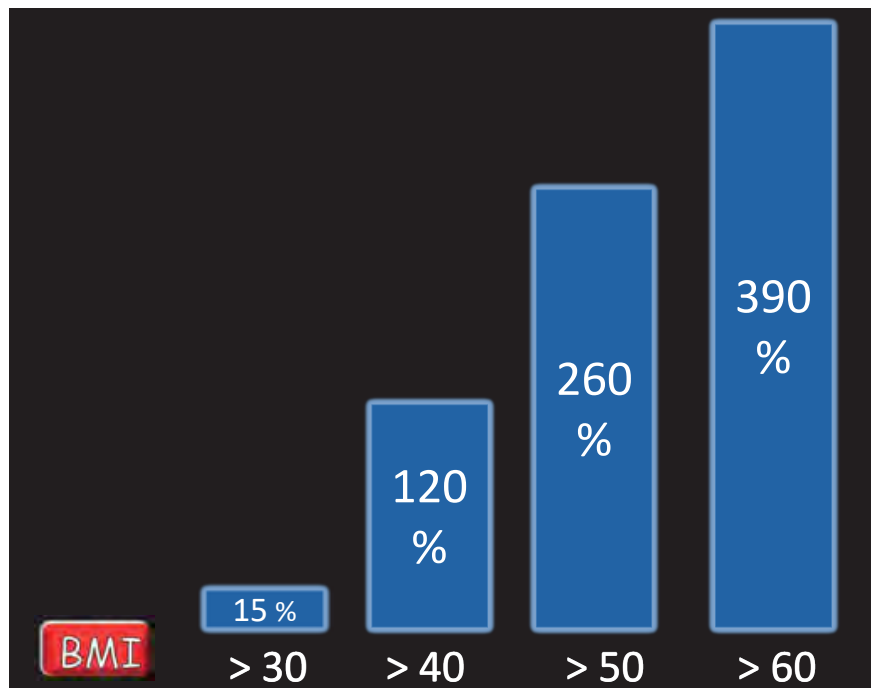

Abb. 1

Ansteigende Prävalenz von Adipositas in der Földiklinik zwischen 2005 und 2015
Lymphödeme“, damals fälschlicherweise auch „Pseudosarkome“ genannt $(5,6,7)$. Diese reduzierte Betrachtungsweise ist erstaunlich, wenn man berücksichtigt, dass Lymphödeme, die im Zusammenhang mit Adipositas stehen, in den vergangenen Jahren massiv zugenommen haben.

Die Földiklinik in Hinterzarten bei Freiburg ist die weltweit größte lymphologische Fachklinik. Patienten, bei denen das Lymphödem oder das Lipödem in Zusammenhang mit der Adipositas steht, bilden inzwischen sowohl in der kassenärztlichen lymphologischen Ambulanz in der Földiklinik als auch stationär die größte und am schnellsten anwachsende Patientengruppe.

Abbildung 1 zeigt ansteigende Prävalenz von Adipositas in der Földiklinik zwischen 2005 und 2015.

- Abbildung 1 zeigt, dass es nur einen milden Anstieg von Patienten mit einem BMI zwischen 30 und $40 \mathrm{~kg} / \mathrm{m}^{2}$ in diesen 10 Jahren gab, aber eine exponentielle $\mathrm{Zu}$ nahme an Patienten mit einem BMI über $40 \mathrm{~kg} / \mathrm{m}^{2}$. Die Földiklinik behandelt ca. 6000 Patienten im Jahr (ambulant 4000, stationär 2000). 2015 waren $66 \%$ unserer Patienten adipös $\left(\mathrm{BMI}>30 \mathrm{~kg} / \mathrm{m}^{2}\right) ; 38 \%$ aller unserer Patienten waren morbid adipös $\left(\mathrm{BMI}>40 \mathrm{~kg} / \mathrm{m}^{2}\right)$. Damit wird auch die hohe absolute Zahl an schwer adipösen Patienten deutlich, die jedes Jahr in der Fachklinik für Lymphologie behandelt werden.

Nach unserer Erfahrung steigt etwa ab einem BMI von $40 \mathrm{~kg} / \mathrm{m}^{2}$ das Risiko für die Entwicklung eines Lymphödemes stark an. Voraussetzung hierfür scheint aber zusätzlich eine „primäre Disposition“ für Einschränkungen im Lymphtransport zu sein. Schließlich leidet nicht jeder morbid adipöse Mensch an Lymphödemen. Aber auch bei Patienten mit einem BMI unter $40 \mathrm{~kg} / \mathrm{m}^{2}$ können sich Lymphödeme entwickeln, wenn neben einer primären Disposition auch andere Lymphödem-aggravierende Begleiterkrankungen wie z. B. Diabetes mellitus, Herzinsuffizienz, CVI oder Immobilität vorliegen.

Typische Lymphödemlokalisationen sind die unteren Extremitäten, die Genitalregion sowie die Bauchdecke. $>$ Abbildung 2 zeigt eine Patientin mit einem BMI von $48 \mathrm{~kg} / \mathrm{m}^{2}$ und distal betonten Beinlymphödemen. Abbildung 3 zeigt einen Patienten mit einem BMI von $66 \mathrm{~kg} / \mathrm{m}^{2}$ und 
Lymphödemen in beiden Beinen (distal betont), Lymphödem der Genitalregion sowie der Bauchdecke.

Bei schwer morbid adipösen Patienten $\left(\mathrm{BMI}>50 \mathrm{~kg} / \mathrm{m}^{2}\right.$ in $>$ Abbildung $4 \mathrm{bzw}$. $\mathrm{BMI}>60 \mathrm{~kg} / \mathrm{m}^{2}$ in $>$ Abbildung 5) kann es darüber hinaus auch $\mathrm{zu}$ massiven lokalen Lymphödemen kommen, früher (wie oben dargestellt) fälschlicherweise als „Pseudosarkom" bezeichnet.

\section{Pathophysiologie}

Wie kann man nun diesen Zusammenhang zwischen Adipositas und Lymphödem erklären? Oder anders gefragt: Warum neigen schwer adipöse Menschen so häufig zu Lymphödemen? Aus der klinischen Perspektive betrachtet scheint es offensichtlich, dass es eine Verbindung zwischen diesen häufig gemeinsam auftretenden Phänomenen gibt.

Die enge Verbindung präsentiert sich auch in dem histologischen Bild von Prof. Zöltzer ( Abb. 6). Es zeigt deutlich, wie nahe Lymphgefäße (hier der Präkollektor) und Fettzellen beieinanderliegen.

Während die Zunahme von viszeralem oder abdominellem Fettgewebe bekanntermaßen stark mit Insulinresistenz und kardiovaskulären Erkrankungen korreliert (9), hat die Zunahme von subkutanem Fettgewebe nicht die gleichen negativen Auswirkungen auf unseren Metabolismus - allerdings, und das ganz erheblich, auf die Funktion unseres Lymphgefäßsystems.

Wir wissen inzwischen, dass Lymphgefäße von subkutanem Fettgewebe umgeben sind (10). Dieses perilymphatische Fettgewebe ist - im Sinne einer Energiequelle metabolisch essenziell sowohl für die lymphatische als auch für die immunologische Funktion des Lymphgefäßsystems (11). Die Zunahme von Fettgewebe im Sinne einer Adipositas führt allerdings über die $\mathrm{Zu}$ nahme der Sekretion von Fettgewebshormonen (Adipokine) zu einer lokalen chronischen Inflammation des perilymphatischen Fettgewebes. Adipokine wie Leptin, TNF-alpha oder HIF1-alpha haben hier einen proinflammatorischen und damit lymphgefäßschädigenden Effekt, während Adiponektin einen lymphgefäßprotektiven Charakter zeigt (78).

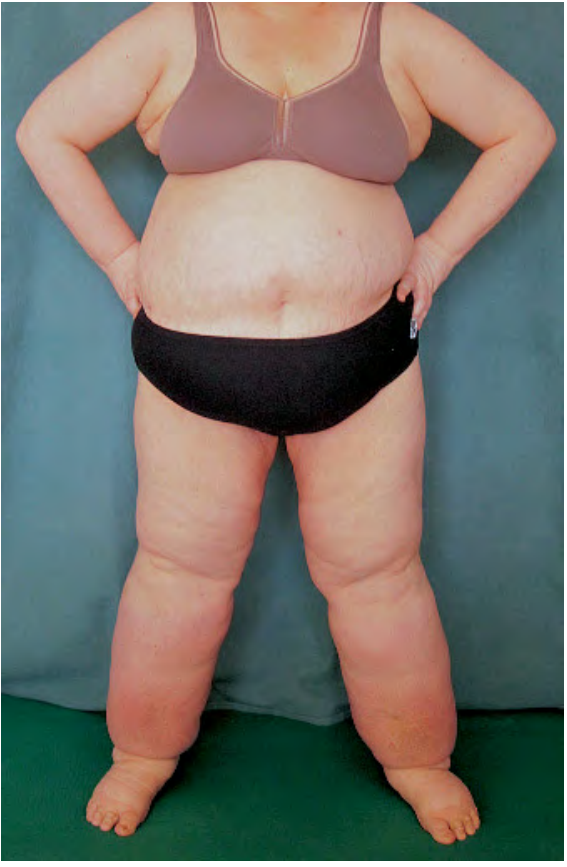

Abb. 2 Patientin mit einem BMl von $48 \mathrm{~kg} / \mathrm{m}^{2}$ und distal betonten Beinlymphödemen

Rutkowksi zeigte, dass diese lokale Inflammation des perilymphatischen Fettgewebes zu einer lymphatischen Leckage führen kann, mit der Konsequenz des Austretens von Lymphe aus dem Lymphgefäß (12).

Andere Studien bestätigen, dass Adipositas sowohl zu einer Beeinträchtigung des Lymphtransports als auch zu einer Verminderung der Aufnahme von Lymphe in den Lymphknoten führt $(13,14)$. Weitmann et al konnten nachweisen, dass Adipositas zu einer Veränderung der Lymphknotenarchitektur führt: so zeigten die untersuchten Lymphknoten eine veränderte reduzierte - Binnenstruktur; gleichzeitig waren auch Größe und die Anzahl der Lymphknoten vermindert (15).

Blum et al konnten sowohl eine reduzierte Frequenz der Muskelkontraktion des Lymphkollektors („Lymphangiomotorik“) als auch eine verringerte Antwort auf Mechanostimulation des Lymphgefäßes bei adipösen Mäusen nachweisen. Hierdurch war auch der Lymphtransport erheblich beeinträchtigt (16).

Gleichzeitig wissen wir von Untersuchungen von E. Földi aus den 80er Jahren (17) sowie von Zampell (18) aus neuerer Zeit, dass auch eine bidirektionale Verbin-

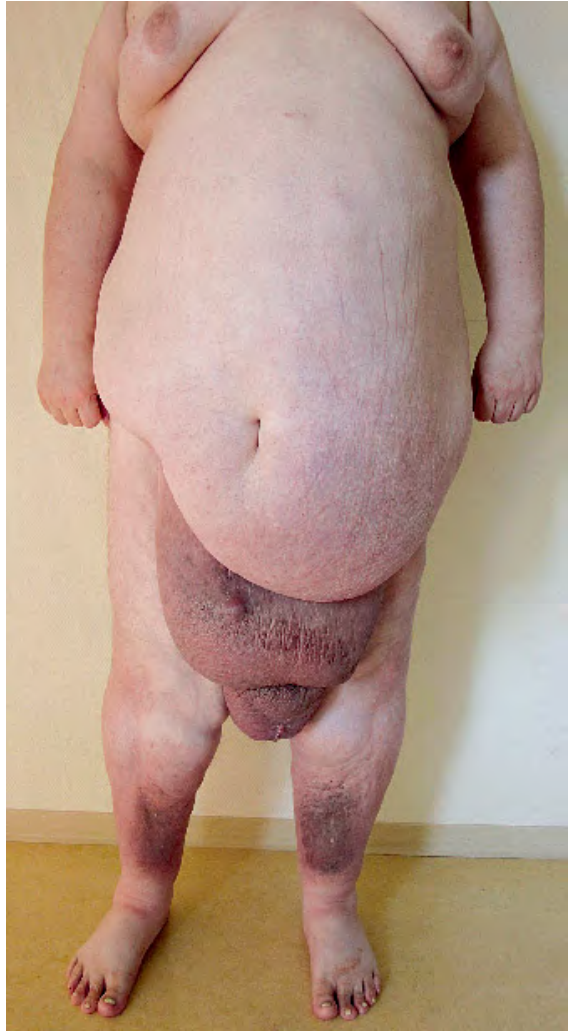

Abb. 3 Patient mit einem BMl von $66 \mathrm{~kg} / \mathrm{m}^{2}$ und Lymphödemen in beiden Beinen (distal betont), Lymphödem der Genitalregion sowie der Bauchdecke.

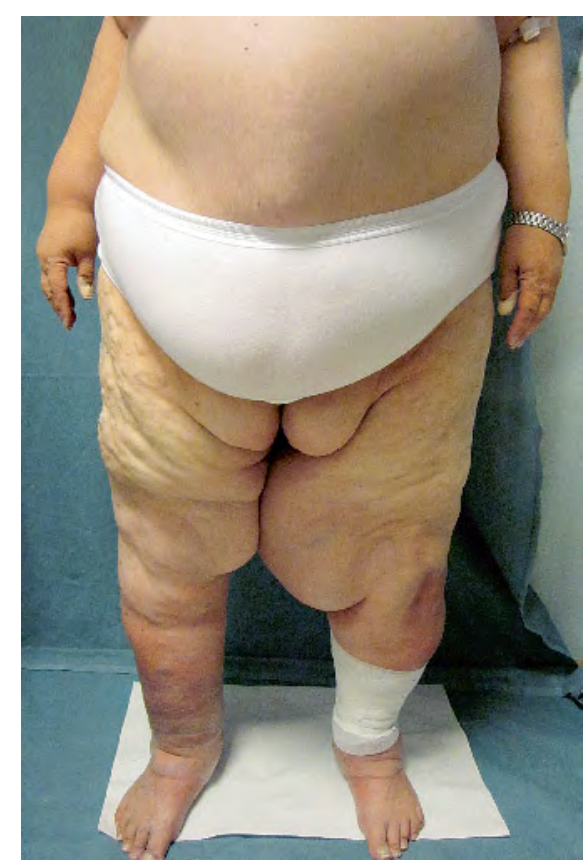

Abb. 4 Schwer morbid adipöser Patient (BMI > $50 \mathrm{~kg} / \mathrm{m}^{2}$ ) 


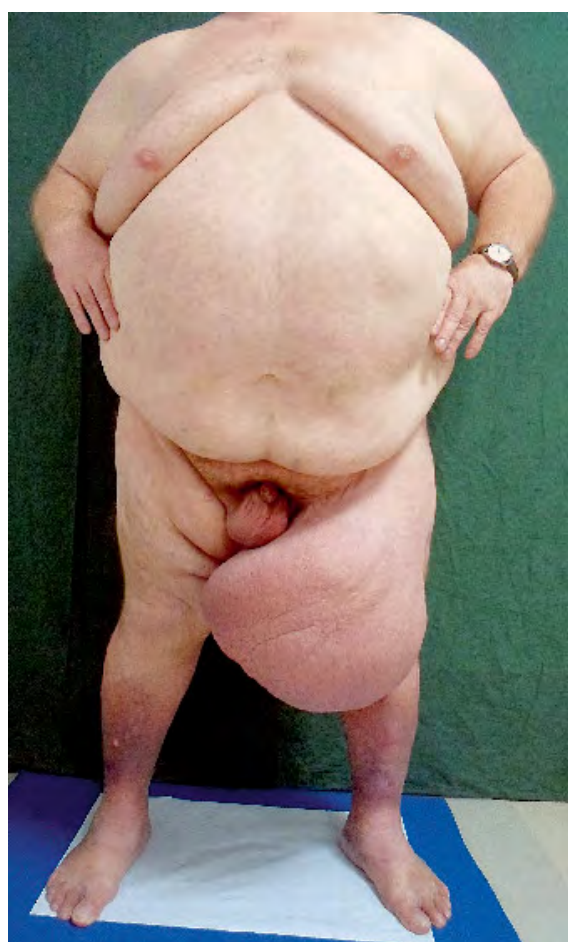

Abb. 5 Schwer morbid adipöser Patient (BMI > $\left.60 \mathrm{~kg} / \mathrm{m}^{2}\right)$

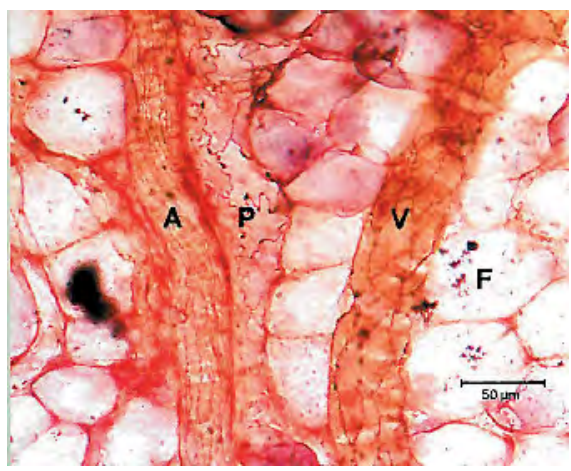

Abb. 6 Lymphgefäße (hier der Präkollektor) und Fettzellen liegen nah beieinander (Prof. Hellmuth Zöltzer in Lymph Forsch 2008 [A= Arterie, $\mathrm{P}=$ Präkollektor, $\mathrm{V}=$ Vene, $\mathrm{F}=$ Fettzellen]) (8).

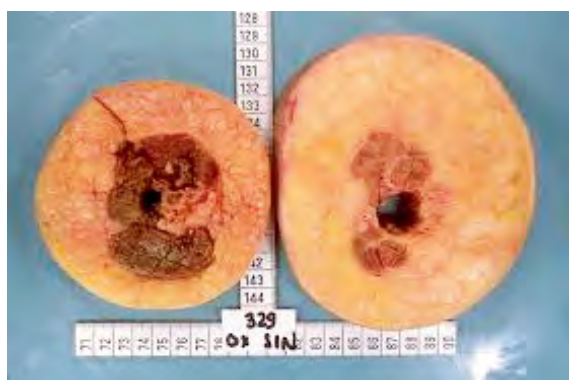

Abb. 7 Lymphödem des rechten Armes (mit freundlicher Genehmigung von Hakan Brorson)

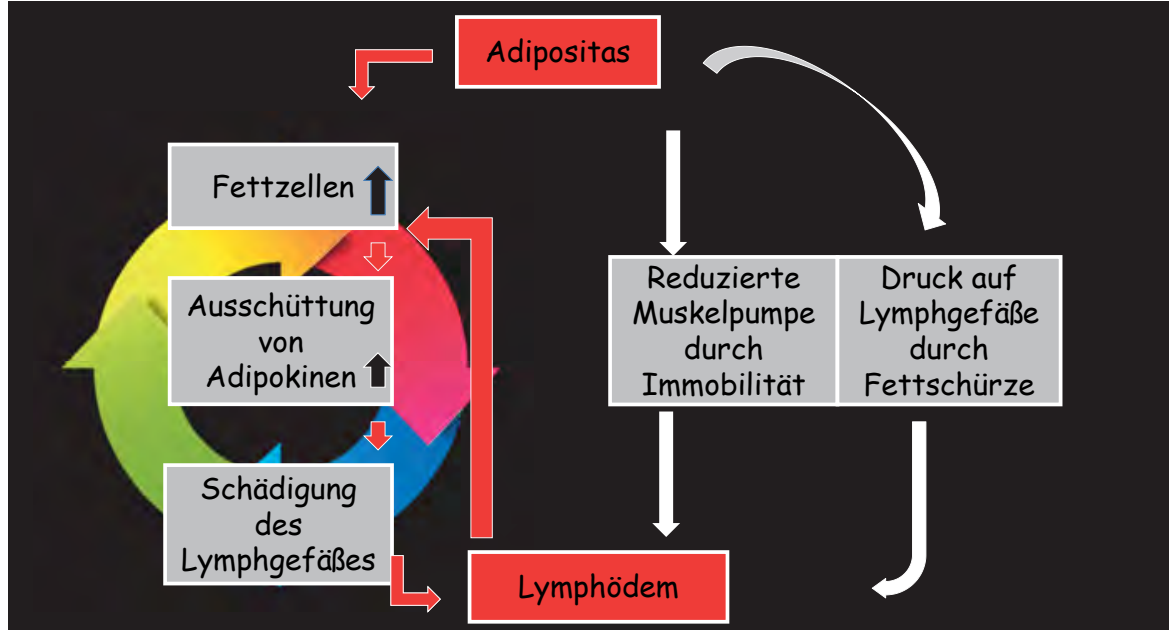

Abb. 8 Die Grafik verdeutlicht die komplexe Ätiologie Adipositas-assoziierter Lymphödeme

dung von Adipositas und Lymphgefäßschädigung existiert. Beide führten Ablationen von Lymphgefäßen bei Kaninchen bzw. bei Mäusen durch. Folge dieser Prozedur war nicht nur ein Lymphödem, sondern auch eine Zunahme an subkutanem Fettgewebe in der Lymphödemregion. Dies wird auch in der Abbildung 7 eines Lymphödemes des rechten Armes deutlich.

Diese - lymphostaseinduzierte - Fettgewebszunahme sezerniert nun wiederum vermehrt Adipokine, die das Lymphgefäß weiter schädigen können. Es handelt sich somit um einen Circulus vitiosus.

Schließlich führen aber auch mechanische Einflussfaktoren zu einer Beeinträchtigung des Lymphtransports. Morbid adipöse Patienten leiden oft an einer verminderten Mobilität. Diese Mobilität ist aber durch den notwendigen Einsatz der Muskelpumpe essenziell für den Lymphtransport. Darüber hinaus präsentieren schwer adipöse Menschen häufig eine herabhängende Bauchfettschürze. Diese kann Lymphgefäße in den Leisten - wie auch Venen (19) - komprimieren, was den Lymphtransport zusätzlich beeinträchtigt.

Verdeutlicht werden diese Zusammenhänge in Abbildung 8 , wobei der bunte Kreis den oben beschriebenen Circulus vitiosus darstellen soll.

\section{Therapie}

Bei der Therapie des Adipositas-assoziierten Lymphödems müssen wir auf zwei Krankheiten fokussieren: einerseits das Lymphödem selbst und andererseits die Adipositas als Ursache des Lymphödemes (bzw. als Ursache der Verschlechterung des Lymphödems).

\section{Krankheit: Lymphödem}

Goldstandard zur Behandlung des Lymphödemes ist die „Komplexe Physikalische Entstauungstherapie“ (KPE). Diese besteht aus folgenden aufeinander abgestimmten Komponenten:

- Hautpflege und falls erforderlich Hautsanierung

- Manuelle Lymphdrainage, bei Bedarf ergänzt durch additive manuelle Techniken

- Kompressionstherapie mit speziellen mehrlagigen, komprimierenden Wechselverbänden und/oder lymphologischer Kompressionsstrumpfversorgung (in der Regel Flachstrickmaterial)

- Entstauungsfördernde Sport-/Bewegungstherapie

- Aufklärung und Schulung zur individuellen Selbsttherapie (20).

Dieses seit Jahrzehnten erfolgreich durchgeführte Konzept zur Behandlung des Lymphödems ist weitestgehend unstrittig.

\section{Krankheit: Adipositas}

Weitaus weniger Konsens besteht in der Frage, wie mit der zweiten Erkrankung, der Adipositas, im Kontext des Lymphödems umgegangen wird.

Hier zeichnen sich zwei Vorgehensweisen ab: 


\section{Negieren oder Ignorieren der Krankheit Adipositas}

In der Praxis wird diese Strategie häufig durch die Diagnose „Lipolymphödem“ ermöglicht. Unter dieser Diagnose sehen wir täglich adipöse Patienten mit einem BMI über 40 , 50 oder gar $60 \mathrm{~kg} / \mathrm{m}^{2}$, die uns ambulant zu- bzw. stationär eingewiesen werden. Hinter dem Konzept „Lipolymphödem" steckt die Vorstellung, dass die Erkrankung Lipödem (über das Lipödem wird in einem anderen Artikel in dieser Zeitschrift referiert) über eine - quasi unkontrollierbare - Fettgewebsvermehrung zur Gewichtszunahme führt. Durch diese Fettgewebsvermehrung soll es dann - nach Ansicht vieler lymphologisch Tätiger - zu einer mechanischen Komprimierung der Lymphgefäße mit der Folge eines Lymphödems kommen $(21,22)$.

Es muss betont werden, dass es für diese weit verbreitete Betrachtungsweise keine wissenschaftliche Evidenz gibt. Trotzdem hat sich diese Sicht in Patientenkreisen als auch unter vielen medizinisch Tätigen breit durchgesetzt. Die Diagnose „Lipolymphödem" ist inzwischen in unserer kassenärztlichen lymphologischen Ambulanz in der Földiklinik (jährlich sehen wir hier ca. 4000 Patienten) die mit Abstand häufigste zugewiesene Fehldiagnose.

In diesem Zusammenhang sei erwähnt, dass die Diagnose „Lipolymphödem“ ohnehin ein wenig sinnvolles Wortkonstrukt ist. „Lipolymphödem“ suggeriert, dass das Lipödem zum Lymphödem führt, es suggeriert, dass das Lipödem Ursache des Lymphödems ist. In manchen Klassifikationen wird das "Lipolymphödem“ sogar als Lipödem im Stadium IV beschrieben $(23,24)$.

Für diese populäre Sichtweise fehlt allerdings ebenfalls jegliche Evidenz. Tatsächlich stellt sich unsere täglich erlebte klinische Praxis ganz anders dar: die bereits adipöse Lipödempatientin mit einem BMI von (z.B.) $35 \mathrm{~kg} / \mathrm{m}^{2}$ nimmt im Verlauf von wenigen Jahren weitere (z. B.) 20 oder 40 kg zu. Folge kann dann - additiv zum Lipödem - auch ein Lymphödem sein. Hierbei handelt es sich jedoch nach unserer Überzeugung nicht um ein Lipödem-induziertes Lymphödem, sondern vielmehr um ein Adipositas-induziertes Lymphödem. Die Pathophysiologie hierfür wurde oben dar- gestellt. Diese Patientin leidet somit an drei unterschiedlichen Erkrankungen: der morbiden Adipositas, dem Lipödem und einem Adipositas-assoziierten Lymphödem. Diese drei Erkrankungen treten in unserem Patientengut gehäuft gemeinsam auf. Der Begriff „Lipolymphödem“ sollte daher m. E. aus der lymphologischen Terminologie getilgt werden. In der Földiklinik benutzen wir diesen Begriff seit Jahren nicht mehr. - Abbildung 9 zeigt eine Patientin mit morbider Adipositas, Adipositas-assoziiertem Lymphödem und Lipödem. Darüber hinaus leidet diese Patientin auch an einer CVI, einem Diabetes mellitus sowie einer arteriellen Hypertonie.

\section{Behandlung der Adipositas mit "Diet and exercise"}

Der übliche Rat, der adipösen Patienten meist mitgegeben wird ist ,weniger zu essen (oder eine Diät zu machen) und sich mehr zu bewegen".

Ernährungsmediziner und Diätassistenten formulieren diese Empfehlung gerne differenzierter und daher auch komplizierter. In den Leitlinien der Deutschen Adipositas-Gesellschaft (DAG) liest man: „Die Behandlung zielt auf eine langfristige Lebensstiländerung mit energieärmerer Kost und Steigerung der körperlichen Aktivität hin" (25). Der von adipösen Patienten erlebte Langzeit-Nutzen dieser Empfehlung bleibt in der Regel aus. In den Leitlinien der DAG werden darüber hinaus verschiedene Gewichtsreduktionsprogramme empfohlen. Konkret werden dort kommerzielle Programme wie "Mobilis", „weight watchers", „Abnehmen mit Genuss" und andere mehr genannt. Wesentlicher Kern aller dort genannten Programme ist immer irgendeine Form von Diät, euphemistisch auch „Ernährungsumstellung " genannt.

Wirft man einen Blick auf die „DiätHistorie" der vergangenen Jahrzehnte fällt auf, dass das Kriterium, ab wann eine Diät als erfolgreich zu bewerten ist, immer weiter aufgeweicht wurde. Nachdem festgestellt wurde, dass eine Gewichtsabnahme von $20 \%$ nur von wenigen Patienten erreicht wurde, senkte man dieses Kriterium erst auf $10 \%$ und zuletzt auf $5 \%(26,27$, 28). Die äußerst bescheidenen „Therapieerfolge" dieser kommerziellen Gewichtsre-

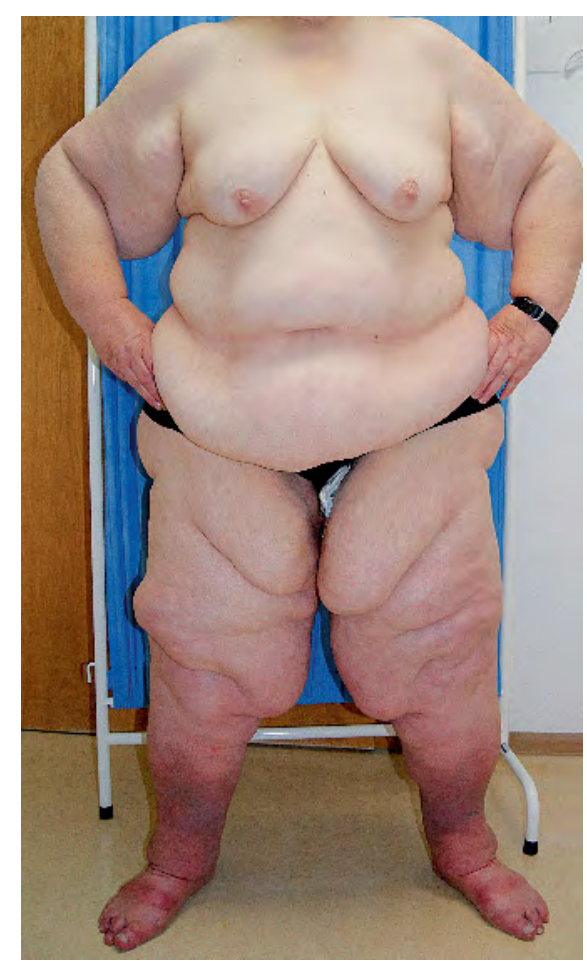

Abb. 9 Patientin mit morbider Adipositas, Adipositas-assoziiertem Lymphödem und Lipödem

duktionsprogramme sind auch in den aktuellen AWMF Leitlinien zur Prävention und Therapie der Adipositas einzusehen (29).

Obwohl Ernährungswissenschaftler - in engem Verbund mit der Diätindustrie - das Erfolgskriterium für Diäten immer weiter heruntergedrückt haben, sind die Patienten diesen Weg nicht mitgegangen. Nicht ein einziger Diätteilnehmer - in einer Untersuchung mit 130 Teilnehmern - wäre mit einem Abnehmerfolg von 5\% zufrieden (30). Diese Diskrepanz zwischen verkauftem Erfolg und gefühltem Erfolg wird auch in weiteren Studien bestätigt (31).

Ein weiterer Grund, Diäten bzw. Gewichtsreduktionsprogrammen kritisch reserviert gegenüberzustehen, ist das in vielen Untersuchungen dokumentierte Scheitern im Langzeitverlauf.

Schaut man sich die wissenschaftliche Evidenz der in den AWMF-Leitlinien zur Adipositastherapie genannten Programme an, stellt man schnell fest, dass ein zufriedenstellender Abnehmerfolg lediglich für das erste Jahr dokumentiert werden kann. Nicht selten werden diese „Ein-Jahres-Studien“ auch als Langzeiterfolg bzw. als „dau- 
erhafte Gewichtsabnahme“ verkauft (32). In der Tat stellt sich aber die Frage, warum trotz seit vielen Jahren existierenden Diätprogrammen wie weight watchers u.a. kaum fundierte Langzeitdaten vorliegen. Die wenigen Langzeitstudien zu Diätverläufen, die es gibt, präsentieren ein erschütterndes Bild (33-40).

Je nach Untersuchung nehmen zwischen $80 \%$ und $99 \%$ der Teilnehmer an Diätprogrammen das abgenommene Gewicht wieder $\mathrm{zu}$ - viele wiegen sogar mehr als vor Beginn der Gewichtsabnahme (41). Metaanalysen zeigen, dass Normalgewichtige, die „diäten“, sogar ein höheres Risiko aufweisen, adipös zu werden als die Vergleichsgruppe, die darauf verzichtet hat (42). Um den möglichen genetischen Einfluss dieser Studie zu berücksichtigen, wurden in einer schwedischen Studie 4000 Zwillinge über 9 Jahre verfolgt. Auch hier zeigte sich, dass „diäten“ das Risiko übergewichtig zu werden nicht vermindert sondern erhöht (43).

Auch wenn diese Fakten - wenn ausgesprochen - regelhaft einen Aufschrei von Diätassistenten und Ernährungsmedizinern zur Folge haben, bleiben sie dennoch Fakten - Fakten, für die eine bestechende Evidenz existiert.

Die Gründe für das Scheitern von Diäten bzw. von Ernährungsmedizin sind komplex, inzwischen aber gut bekannt. Eine Darstellung der umfangreichen physiologischen Prozesse, die nach erfolgter Gewichtsabnahme wieder zu einer Gewichtszunahme führen, würde den Rahmen des vorgegebenen Themas sprengen. Es gibt jedoch eine gute Evidenz, dass sowohl endokrinologische, psychologische, metabolische und neurophysiologische Veränderungen, die während einer Gewichtsabnahme auftreten, erheblichen Anteil an diesem Prozess haben (45-48). Darüber hinaus leben wir seit wenigen Jahrzehnten in einer Umwelt, die ganz wesentlich zum Übergewicht beiträgt („obesigenic environment)“(49).

Fasst man die historische und die aktuelle Studienlage zusammen, so verwundert es doch sehr, dass der überwältigende Teil der Ärzteschaft adipöse Patienten weiter zum Abnehmen drängt. Es ist evident, dass Patienten damit eher auf einen Weg geführt werden, der im Langzeitverlauf zu weiterer Gewichtszunahme führt.

\section{Adipositaskonzept in der Földiklinik}

Die oben beschriebene Datenlage deckt sich auch mit unserer täglichen klinischen Erfahrung mit adipösen Patienten. Abnehmen schaffen alle, Gewicht halten nur sehr wenige! Wir haben daher unseren therapeutischen Ansatz zur Behandlung von adipösen Patienten mit Lymphödemen oder mit Lipödemen modifiziert. Unser therapeutischer Ansatz besteht aus fünf Säulen, die hier kurz skizziert werden.

\section{Auf die Adipositas fokussieren statt sie ignorieren!}

Die Rolle der Adipositas wird von uns als wesentlicher Faktor anerkannt, der zur Entstehung oder zur Verschlechterung des Lymphödems aber auch des Lipödems beiträgt. Die alleinige Fokussierung auf die Behandlung des Lymphödems (oder des Lipödems) ist nicht zielführend und führt allenfalls zu einer kurzzeitigen Verbesserung der lymphologischen Erkrankung.

\section{Adipositas als Krankheit anerkennen!}

Adipositas ist eine - übrigens auch von der WHO sowie der American Medical Association anerkannte - Erkrankung (50, 51). Diese essenzielle Haltung gegenüber Patienten spiegelt sich auch in der Wahrnehmung der Adipositas bei unseren medizinisch, therapeutisch oder psychologisch Tätigen in der Földiklinik wider. Adipositas wird von uns nicht als schuldhaftes Verhalten, nicht als Folge von Willensschwäche oder Disziplinlosigkeit begriffen, sondern als eine Krankheit, die multiple und sehr komplexe Ursachen hat. Damit ist nicht gesagt, dass adipöse Menschen ihrer Erkrankung hilflos ausgeliefert sind. Aber es geht um eine veränderte Grundhaltung, eine Grundhaltung, die sich wegbewegt von Begriffen wie Schuld oder „sich gehen lassen“ hin zu Respekt und Verständnis für die Situation des anderen. Diese veränderte - von Respekt und Empathie getragene Haltung unserer Mitarbeiter wird von unseren Patienten auch dankbar wahrgenommen.

\section{Veränderung der therapeutischen Ziele}

Die oft reflexartige Fokussierung auf die Gewichtsabnahme von übergewichtigen Patienten suggeriert, dass Übergewicht und Adipositas zwangsläufig mit einer erhöhten Sterblichkeit verknüpft sind. Tatsächlich ist die Datenlage, ab welchem BMI ein erhöhtes Mortalitätsrisiko vorliegt, nicht konsistent. Während manche Untersuchungen dieses Risiko bereits für einen BMI ab 25 $\mathrm{kg} / \mathrm{m}^{2}$ als erhöht betrachten, sehen andere Studien das Mortalitätsrisiko erst ab einem BMI ab $30 \mathrm{~kg} / \mathrm{m}^{2}$, bzw. $35 \mathrm{~kg} / \mathrm{m}^{2}$ als signifikant erhöht (52-54).

Viel überzeugender - und konsistenter - ist die Studienlage bei der Frage, welche Variablen tatsächlich eine verfrühte Letalität voraussagen. Adipositas findet sich hier nicht unter den Top 5. „Schlechte Fitness“, „Rauchen“, „Bluthochdruck“, „Niedriges Einkommen" und „Einsamkeit" waren einzeln betrachtet jeweils bessere Prädiktoren für ein erhöhtes Sterblichkeitsrisiko als Übergewicht oder Adipositas (55-57).

Aus diesem Grunde haben wir unsere Therapieziele bei unseren übergewichtigen und adipösen Patienten in der Földiklinik verändert. Statt auf Gewichtsabnahme fokussieren wir auf Gewichtsstabilität, statt auf Gewichtsabnahme fokussieren wir auf tägliche Bewegung und gesundes Essen, Fitness und Lebensqualität.

Statt „diet and exercise“ ist unser Credo „stabilize and exercise“!

\section{Gewichtsreduktionsprogramme sind nicht Lösung des Problems Adi- positas - sie sind Teil des Problems.}

Aus dem oben Gesagten folgt, dass wir Patienten über die tatsächlichen - und frustranen - Erfolgsaussichten von Diäten informieren. Viele gerade unserer schwer adipösen Patienten haben eine Jahrzehnte andauernde "Diätkarriere" hinter sich mit der Folge einer steten Gewichtszunahme (,weight cycling"). Aufgrund der vorliegenden und oben genannten Datenlage, die sich darüberhinaus auch in der Biografie unserer Patienten widerspiegelt, raten wir unseren Patienten strikt von Diäten ab. Wir betrachten Diäten und Gewichtsreduktionsprogramme als ein Geschäftsmodell, 
dass lediglich einen ökonomischen Nutzen für die Diätindustrie - und damit „verbandelte“ Ärzte hat $(79,80)$.

Statt den Fokus auf Gewichtsabnahme und Diäten zu legen, werden Patienten lediglich über gesundes Essen beraten. Schwerpunkt hier ist die Aufklärung über den inzwischen gut belegten Zusammenhang zwischen Zucker (in all seinen verschiedenen Facetten und Verkleidungen) und Gewichtszunahme (58-62). Ein weiteres Hauptanliegen besteht darin, Patienten für Bewegung $\mathrm{zu}$ begeistern. Neben dem Ziel einer langfristigen Gewichtsstabilisierung und einer verbesserten Fitness ist die Bewegungstherapie auch Teil der komplexen physikalischen Entstauungstherapie zur Behandlung des Lymphödems.

\section{Metabolische Chirurgie bei schwer adipösen Patienten}

$\mathrm{Ab}$ einem BMI von $40 \mathrm{~kg} / \mathrm{m}^{2}$ (bzw. BMI 35 $\mathrm{kg} / \mathrm{m}^{2}$ bei gleichzeitig bestehenden schweren internistischen Begleiterkrankungen) überprüfen wir, ob der Patient von einem adipositaschirurgischen Eingriff profitieren könnte. Seit 2009 bieten wir in der Földiklinik ein multimodales Adipositasprogramm an, in dessen Zentrum der bariatrische Eingriff steht. Dieses Programm umfasst ein umfangreiches internistisches, endokrinologisches, chirurgisches und psychologisches Assessment. Darüber hinaus enthält es Module, in denen sowohl die Bedeutung von täglicher Bewegung vermittelt wird als auch eine ernährungsmedizinische Begleitung, die den postoperativen Kostaufbau sowie die Notwendigkeit der Supplementation erklärt. Jede Woche findet ein kleines „Adipositas-Board“ statt, an dem ein Internist (mit Zusatzqualifikation in Adipositasmedizin "SCOPE"), eine Diabetologin und eine auf diese Patientengruppe spezialisierte Diplom-Psychologin (Psychologische Psychotherapeutin) teilnehmen. Bevor wir den Patienten dem Adipositaschirurgen zur Begutachtung vorstellen, wird seine „OP-Eignung" in diesem Board internistisch und psychologisch überprüft. Etwa ein bis zwei Jahre nach bariatrischer OP erfolgt, falls notwendig, die plastische OP zur Resektion der überschüssigen Hautlappen. Das Programm ist langfristig angelegt; so sehen wir die Patienten ca. alle 9 Monate in
Abb. 10

Viele Berufsgruppen im Adipositasprogramm ziehen gemeinsam an einem Strang.

unserer kassenärztlichen lymphologischen Ambulanz - bei Bedarf auch früher.

- Abbildung 10 zeigt, wie viele Berufsgruppen in unserem Adipositasprogramm gemeinsam an einem Strang ziehen.

Die Studienlage zur Adipositaschirurgie ist bestechend. Es bestehen multiple Langzeitdaten, die den Erfolg dieser Therapieoption nach 5 Jahren, nach 7 Jahren, nach 15 Jahren oder auch nach 20 Jahren bestätigen. (63-68). Die positiven Auswirkungen einer anhaltenden Gewichtsabnahme im Rahmen eines bariatrischen Eingriffes auf das Lymphödem - aber auch auf das Lip-

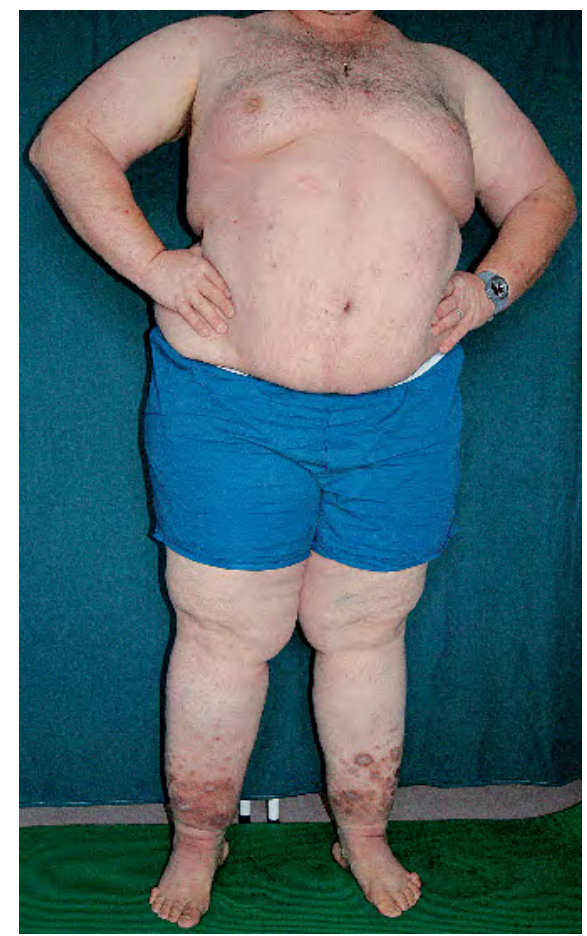

Abb. 11 Patient mit Adipositas-assoziierten Phlebolymphödemen - vor Teilnahme am Adipositasprogramm der Földiklinik ödem - sehen wir seit 2009. Aktuell läuft eine Studie mit dem Universitätsklinikum Freiburg, die auch wissenschaftlichen Daten zu dieser Vorgehensweise liefern soll.

- Abbildungen 11 und 12 zeigen einen Patienten mit Adipositas-assoziierten Phlebolymphödemen - ca. 2 Jahre nach Teilnahme am Adipositasprogramm in der Földiklinik. Der BMI bei Erstaufnahme in der Földiklinik betrug $56 \mathrm{~kg} / \mathrm{m}^{2}$. Nach Vorbereitung auf den adipositaschirurgischen Eingriff erfolgte 4 Monate nach Entlassung die Magen-Bypass-Operation. Weitere 18 Monate später wurde die plastisch-chirur-

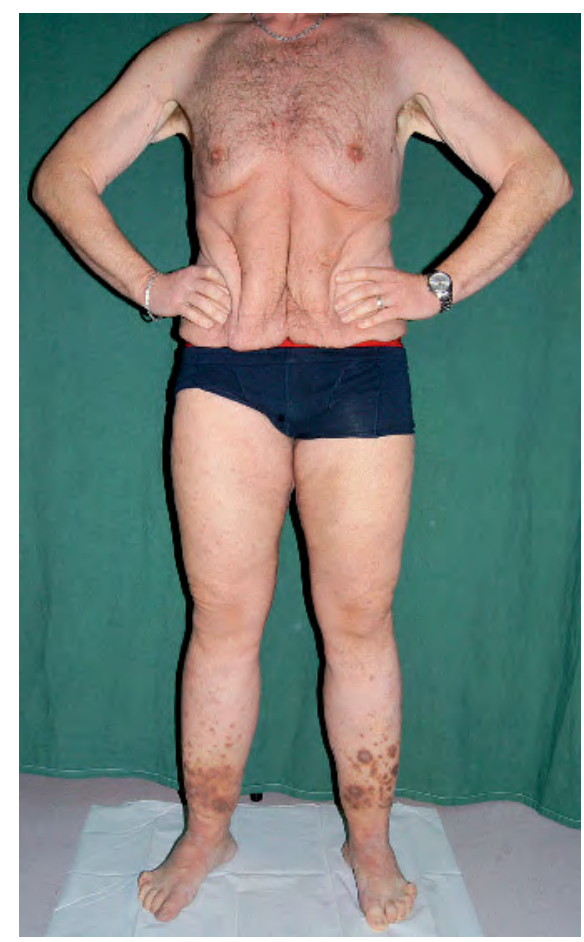

Abb. 12 Patient mit Adipositas-assoziierten Phlebolymphödemen - ca. 2 Jahre nach Teilnahme am Adipositasprogramm der Földiklinik 


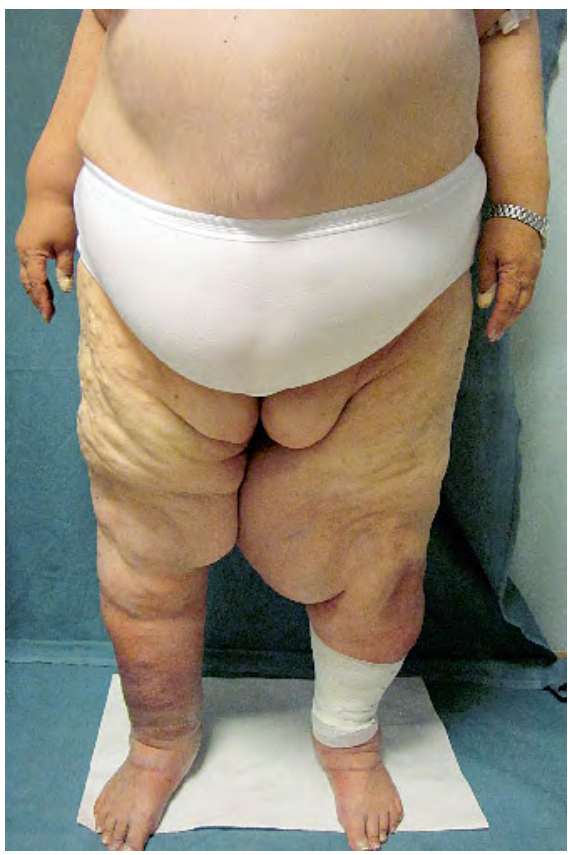

Abb. 13 Patientin mit Adipositas-assoziierten Beinlymphödemen bei gleichzeitigem ausgeprägtem lokalisiertem Lymphödem des linken Oberschenkels vor Teilnahme am Adipositasprogramm der Földiklinik

gische Hautstraffung beider Oberschenkel durchgeführt. Eine Abdominalplastik hat der Patient beantragt; der aktuelle BMI ist $29 \mathrm{~kg} / \mathrm{m}^{2}$. Die deutliche Verbesserung der Beinlymphödeme (Phlebolymphödeme) ist gut darstellbar.

- Abbildungen 13 und 14 zeigen eine Patientin mit Adipositas-assoziierten Beinlymphödemen bei gleichzeitigem ausgeprägtem lokalisertem Lymphödem des linken Oberschenkels vor und ca. 2 Jahre nach Teilnahme am Adipositasprogramm in der Földiklinik. Der BMI bei Erstaufnahme betrug $52 \mathrm{~kg} / \mathrm{m}^{2}$. Nach Schlauchmagen-OP und Gewichtsstabilisierung bei einem BMI von $31 \mathrm{~kg} / \mathrm{m}^{2}$ wurde eine Resektion der Hautlappen beider Oberschenkel vorgenommen. Darüber hinaus erhielt die Patientin eine Bauchdeckenstraffung mit invertiertem T-Schnitt. Auch hier zeigt sich eine massive Verbesserung des lymphologischen Befundes.

\section{Fazit}

Adipositas-assoziierte Lymphödeme stellen eine zunehmende Herausforderung inner-

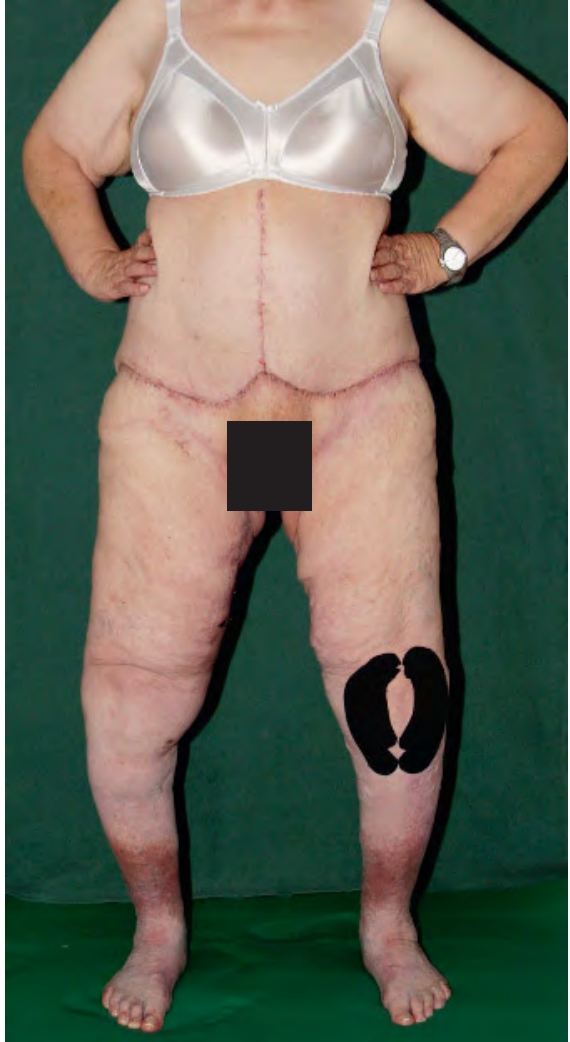

Abb. 14 Patientin mit Adipositas-assoziierten Beinlymphödemen bei gleichzeitigem ausgeprägtem lokalisiertem Lymphödem des linken Oberschenkels ca. 2 Jahre nach Teilnahme am Adipositasprogramm der Földiklinik

halb der Lymphologie dar. Eine auch langfristig erfolgreiche Behandlung ist nur dann möglich, wenn die Adipositas als wesentliche Ursache des Lymphödemes anerkannt und mitbehandelt wird. Von kommerziellen Diäten und Gewichtsreduktionsprogrammen sollte Patienten aufgrund der nachgewiesenen Erfolglosigkeit strikt abgeraten werden. Bei morbid adipösen Patienten ist die metabolische Chirurgie eine wichtige Therapieoption. Diese sollte allerdings in ein multimodales und langfristig angelegtes Therapiekonzept eingebunden sein.

\section{Danksagung}

Mein besonderer Dank gilt Frau Prof. E. Földi für persönliche und fachliche Unterstützung.

\section{Interessenkonflikt}

Nach Angaben des Autors bestehen keine Interessenkonflikte.

\section{Ethische Richtlinien}

Für das Manuskript wurden keine Studien an Menschen oder Tieren durchgeführt.

\section{Literatur}

1. WHO. Controlling the global obesity epidemic, 2017, verfügbar unter http://www.who.int/nutri tion/topics/obesity/en/

2. Robert Koch-Institut 2016, Studie DEGS1, Erhebung 2008-2011.

3. Sifferlin A. $40 \%$ of Americans Are Obese - And the Trend Isn't Slowing in TIME HEALTH, Oktober 2017 verfügbar unter http://time.com/ 4980225/obesity-rates-adults-children

4. Gregg EW et al. Global Health Effects of Overweight and Obesity. N Engl J Med 2017; 377: $80-81$.

5. Farshid G, Weiss SW. Massive localized lymphedema in the morbidly obese. Am J Surg Pathol 1998; 22 1277-1283.

6. Wu D et al. Massive localized lymphedema. Hum Pathol 2000; 31: 1162-1168.

7. Vana J et al. Massive localized lymphedema in an extremely obese patient. Chirurg 2002; 73: 383-386.

8. Zoeltzer H, Linker, I. Präkollektor und Randsinus des Lymphknotens. LymphForsch 11 (2) 2007; 76

9. Bergman $\mathrm{RN}$ et al. Why visceral fat is bad: mechanisms of the metabolic syndrome. Obesity (Silver Spring). 2006 Feb;14 Suppl 1: 16-19.

10. Georgescu A et al. Initiale Lymphsinus und Präkollektoren innerhalb der Fettläppchen der Subcutis des Menschen. LymphForsch 2008; 12 (2): 61-70.

11. Ponds CM. PLEFA. Adipose tissue and the immune system 2005; 73(1): 17-30.

12. Rutkowski J et al. Mechanisms of Obesity and Related Pathologies: The Macro- and Microcirculation of Adipose Tissue. FEBS Journal 2009; 276 (20): 5738-5746.

13. Savetsky I et al. Obesity increases inflammation and impairs lymphatic function in a mouse model of lymphedema. Am J Physiol Heart Circ Physiol 2014; 307: H165-H172.

14. Mehrara BJ, Greene AK. Lymphedema and Obesity: Is there a Link? Plastic Reconst Surgery 2014; 134 (1): 154e-160e.

15. Weitmann ES et al. Obesity impairs lymphatic fluid transport and dendritic cell migration to lymph nodes. PLoS One 2013; 12: 8(8).

16. Blum KS et al. Chronic high-fat diet impairs collecting lymphatic vessel function in mice. PLoS One 2014; 9(4).

17. Földi E et al. Adipositas, Lipödem und Lymphostase. Medwelt 1983, 25.

18. Zampell JC et al. Regulation of Adipogenesis by Lymphatic Fluid Stasis Part 1. Plastic Reconstruct. Surgery 2012; 129 (4): 825-834. 
19. Földiklinik Hinterzarten. Eigene Untersuchungen. Unveröffentlicht

20. S2K Leitlinie. Diagnostik und Therapie der Lymphödeme, verfügbar unter http://www.awmf.org/ uploads/tx_szleitlinien/058-0011_S2k_Diagnostik_und_Therapie_der_Lymphoedeme_2017-05.pdf

21. Wiedner $M$ et al. Entstehung des Lipödems. Lymphselbsthilfe 2017, 15-16.

22. Rüger K. Das Adipositas-Lymphödem. LymphForsch 2008; 12 (1): 31-35.

23. Das LipoLymphödem. Verfügbar unter http://www.lymphverein.de/lipoedem.html

24. Buck DW, Herbst KL. Lipedema: A Relatively Common Disease with Extremely Common Misconceptions. Plast Reconstr Surg Glob Open 2016; $4(9)$.

25. S3 Leitlinie zur Prävention und Therapie der Adipositas. 2014. Verfügbar unter http://www.awmf. org/uploads/tx_szleitlinien/050-0011_S3_Adipositas_Pr\%C3\%A4vention_Therapie_2014-11.pdf.

26. Wing RR, Jeffery RW. Outpatient treatment of obesity: A comparison of methodology and clinical results. International J of Obesity 1979; 3: 261-279.

27. Wing RR, Phelan S. Long-Term Weight Loss Maintenance. American Journal of Clinical Nutrition 2005; 82 (1 Suppl): 222S-225S.

28. Institute of Medicine. The Nature and Problem of Obesity in Weighing the Options: Criteria for Evaluating Weight-Management Programs. Washington DC NA Press 1995, 55-58.

29. S3 Leitlinie zur Prävention und Therapie der Adipositas 2014. a.a.O. S. 61.

30. Jeffery RW et al. Are smaller weight losses or more achievable weight loss goals better in the long term for obese patients? Journal of Consulting and Clinical Psychology 1998; 66 (4): 641-645.

31. Foster GD et al. What is a reasonable weight loss? Patients expectations and evaluations of obesity treatment outcomes. Journal of Consulting and Clinical Psychology 1997; 65 (1): 79-85.

32. Saxenda Fachinformation. Adipositas 2017.11.145

33. Stunkard A. The Results of Treatment for Obesity. A Review of the Literature and Report of a Series. AMA Arch Intern Med 1959; 103(1): 79-85.

34. Bennett W, Gurin J. The Dieter's Dilemma: Why Diets Are Obsolete-the New Setpoint Theory of Weight Control. New York: Basic Books, 1982.

35. Cogan J Rothblum E. Outcomes of weight-loss Programms. Genetic, Social and General Psychology Monographs 1993; 118 (4).

36. Perri MG et al. Sucess and failure in the treatment of obesity: Where do we go from here? Medicine, Exercise, Nutrition and Health 1995; 4: 255-272.

37. Hensrud DD, Weinsier RL. A prospective study of weight maintenance in obese subjects reduced to normal body weight without weight-loss training .American clinical Nutrtion 1994; 60(5): 688-694.

38. Mann T, Tomiyama AJ et al. Medicare's search for effective obesity treatments: diets are not the answer. Am Psychol 2007; 62(3): 220-233.

39. Nordmann A et al. Effects of low carb vs low fat diets on weight loss and cardiovascular risk fac- tors: a meta analysis of randomized controlled trials. Arch Internal Med 2006; 166 (8).

40. Fildes A, Charlton J. Probability of an obese person attaining normal body weight. American Journal of Public Health 2015.

41. Dulloo AG et al. How dieting makes the lean fatter. Obesity reviews 2015; 16 (S1): 25-35.

42. Lowe MR. Dieting: Proxy or Cause of Furture weight gain? Obesity Reviews 2015; 16 (S1): 19-24.

43. Pietiläinen KH et al. Does dieting make you fat? A Twin study. International Journal of Obesity 2012; 36 (3): 456-464.

44. Stice E et al. Caloric deprivation increases responsivity of attention and reward brain regions to intake, anticipated intake and images of palatable foods. NeuroImage 2013; 67: 322-330.

45. Sumithran P et al. Long-Term Persistence of Hormonal Adaptions to Weight Loss. N Engl J Med 2011; 365: 1597-1604.

46. Tataranni PA and Ravussin E. in Energy Metabolism and Obesity in Handbook of Obesity Treatment. New York Guilford Press 2004; 42-72.

47. Leibel RI and Hirsch J. Changes in Energy Expenditure Resulting form Altered Body Weight. N Engl J Med 1995; 332: 10.

48. Lipek $\mathrm{T}$ et al. Obesongenic environment: environmental approaches to obesity prevention. J Pediatr Endocrinol Metab 2015; 28(5-6): 485-495.

49. WHO Obesity. Preventing and managing the global epidemic Report of a WHO Consultation on Obesity, 1997, Geneva.

50. Pollack A, A.M.A. Recognizes Obesity as a Disease: New York Times. June 182014.

51. Zheng $\mathrm{H}$ et al. Obesity and mortality risk: new findings from body mass index trajectories. Am J Epidemiol. 2013 Dec 1;178(11) 1591-9

52. Pischon $T$ et al. General and abdominal adiposity and risk of death in Europe. N Engl J Med 2008; 359(20): 2105-2120.

53. Flegal KM et al. Association of all-cause mortality with overweight and obesity using standard body mass index categories: a systematic review and meta-analysis. JAMA 2013; 309(1): 71-82.

54. Blair SN. Physical inactivity: The biggest public health problem oft he 21st century. British Journal of Sports Medicine 2009; 43 (1): 1-2.

55. Lantz $\mathrm{P}$ et al. Socioeconomic and behavioural risk factors for mortality in a national 19 year prospective study of U.S adults. Social Science \& Medicine 2010; 70 (10): 1558-1566.

56. Holt-Lunstad J et al. Social relationships and mortality risk: a metaanalyses review. Plos med 2010; 7.

57. Malik VS et al. Sweeteners and Risk of Obesity and Type 2 Diabetes: The Role of Sugar-Sweetened Beverages. Curr Diab Rep 2012; 12: 195-203.

58. Johnson, RK et al. Dietary sugars intake and cardiovascular health: a scientific statement from the American Heart Association. Circulation. 2009 120(11), 1011-1020

59. AHA. Dietary Sugars Intake and Cardiovascular Health A Scientific Statement from the American Heart Association Circulation. 2009; 120:1011-1020
60. University of California San Francisco. Sugar science. Verfügbar unter http://sugarscience.ucsf.edu/

61. Lustig R. Fat Chance: The Hidden Truth About Sugar, Obesity and Disease 2013.

62. Inge, $\mathrm{TH}$ et al. Long-term outcomes of bariatric surgery in adolescents with severe obesity: a prospective follow-up analysis. Lancet Diabetes Endocrinol. 2017. Volume 5, Issue 3, March 2017, Pages 165-173.

63. Obeid NR et al. Long-term outcomes after Rouxen-Y gastric bypass: 10- to 13-year data. Surg Obes Relat Dis 2016.

64. Sjöstrom, L et al. Effects of bariatric surgery on mortality in Swedish obese subjects. N Engl J Med 2007; 357(8): 741-752.

65. Adams T et al. Health Benefits of Gastric Bypass Surgery after 6 Years. JAMA 2012. Sep 19308 (11) 1122-1131.

66. Adams $\mathrm{T}$ et al. Long-Term Mortality after Gastric Bypass Surgery. N Engl J Med 2007; 357. 753-761

67. Arterburn DE et al. Association between bariatric surgery and long-term survival. JAMA 2015; 313(1): 62-70.

68. Stunkard AJ et al. An Adoption Study of Human Obesity. N Engl J Med 1986; 314: 193-198.

69. Stunkard AJ et al. The Body-Mass Index of Twins who have been reared apart. N Engl J Med 1990; 322: 1483-1487.

70. Plagemann A (Ed.). Perinatal Programming The State of the Art. Berlin/Boston: Walter de Gruyter 2012; 11-22.

71. Herrera B et al. Genetics and epigenetics of obesity. Maturitas 2011; 69 (1): 41-49.

72. Hewagalamulage SD et al. Stress, Cortisol and obesity: a role for cortisol responsiveness in identifying individuals prone to obesity. Domest Anim Endocrinol 2016; 56 Suppl: 112-120.

73. Volkow ND et al. Obesity and addiction: neurobiological overlaps. Obesity reviews 2013 (1): 2-18

74. Nemiary D et al. The Relationship Between Obesity and Depression Among Adolescents. Psychiatr Ann. 2013; 42 (8): 305-308.

75. Andersen RE (Ed.). Obesity - Etiology, Assessment, Treatment and Prevention. Part 1 and 2. Human Kinetics 2003.

76. Taubes G. The Case against Sugar. New York: Alfred A Knopf 2016.

77. Földi M. Földi E (Hrsg.) Lehrbuch Lymphologie. 7. überarbeitete Auflage, München: Urban \& Fischer 2010; 175-238

78. Hongyu $\mathrm{W}$ et al. Independent and Opposite Associations of Trunk and Leg Fat Depots with Adipokines, Inflammatory Markers, and Metabolic Syndrome in Middle-Aged and Older Chinese Men and Women. J Clin Endocrinol Metab, September 2010, 95(9): 4389-4398.

79. S3-LEITLINIE Interdisziplinäre Leitlinie der Qualität S3 zur „Prävention und Therapie der Adipositas“. AWMF-Register Nr. 050/001 Version 2.0 (April 2014). 58-62.

80. Gemeinschaftsproduktion ZDF und ARTE. Doku: planet e.: Schlank durch Schokolade - Der Trick mit den Diäten. 2015. Verfügbar unter: https://www.youtube.com/watch?v=-35dij13n-U. Hier insbesondere Minute 8 bis Minute 14 . 